\title{
Deforming $\mathfrak{h}$-trivial the Lie algebra $\operatorname{Vect}\left(S^{1}\right)$ inside the Lie algebra of pseudodifferential operators $\Psi \mathcal{D O}$
}

\author{
Imed Basdouri*, ${ }^{*}$, Issam Bartouli ${ }^{\dagger, \ddagger}, \boldsymbol{\Phi}$ and Jean Lerbet ${ }^{\dagger, \|}$ \\ *Département de Mathématiques \\ Faculté des Sciences de Gafsa \\ Université de Gafsa, Gafsa, Tunisie \\ †Université Evry Val d'Essonne \\ Évry 91000, France \\ ${ }^{\ddagger}$ Faculté des Sciences, Département de Mathématiques \\ Université de Sfax, Sfax, Tunisie \\ §basdourimed@yahoo.fr \\ $\boldsymbol{\top}_{\text {issambartouli@yahoo.fr }}$ \\ " jean.lerbet@univ-evry.fr
}

\begin{abstract}
In this paper, we consider the action of $\operatorname{Vect}\left(S^{1}\right)$ by Lie derivative on the spaces of pseudodifferential operators $\Psi \mathcal{D O}$. We study the $\mathfrak{h}$-trivial deformations of the standard embedding of the Lie algebra $\operatorname{Vect}\left(S^{1}\right)$ of smooth vector fields on the circle, into the Lie algebra of functions on the cotangent bundle $T^{*} S^{1}$. We classify the deformations of this action that become trivial once restricted to $\mathfrak{h}$, where $\mathfrak{h}=\mathfrak{a f f}(1)$ or $\mathfrak{s l}(2)$. Necessary and sufficient conditions for integrability of infinitesimal deformations are given.
\end{abstract}

Keywords: Deformation theory; cohomology; Lie algebras; Poisson Lie algebra.

Mathematics Subject Classification 2010: 13D10, 13D03, 17B10, 14B15

\section{Introduction}

Deformation theory plays a crucial role in all branches of mathematics and physics. In physics, the mathematical theory of deformations has proved to be a powerful tool in modeling physical reality. We study some related questions of deformations of some $\mathfrak{g}$-modules. Of course, if $\mathrm{M}=\operatorname{End}(V)$, where $V$ is a $\mathfrak{g}$-module then, according to Nijenhuis-Richardson, the space $\mathrm{H}^{1}(\mathfrak{g}$; End $(V))$ classifies the infinitesimal deformations of a $\mathfrak{g}$-module $V$ and the obstructions to integrability of a given infinitesimal deformation of $V$ are elements of $\mathrm{H}^{2}(\mathfrak{g} ; \operatorname{End}(V))$. More generally, if $\mathfrak{h}$ is a subalgebra of $\mathfrak{g}$, then the $\mathfrak{h}$-relative cohomology $\mathrm{H}^{1}(\mathfrak{g}, \mathfrak{h}$; $\operatorname{End}(V))$ measures the infinitesimal $\mathfrak{h}$-trivial deformations that become trivial once the action is restricted to $\mathfrak{h}$ (h-trivial deformations), while the obstructions to extension of any $\mathfrak{h}$-trivial infinitesimal deformation to a formal one are related to $\mathrm{H}^{2}(\mathfrak{g}, \mathfrak{h} ; \operatorname{End}(V))$. 
Deformations of Lie algebras with base and versal deformations were already considered by Fialowski in 1986 [8]. In 1988, Fialowski [9] further introduced deformations whose base is a complete local algebra. Also, in [9], the notion of miniversal (or formal versal) deformation was introduced in general, and it was proved that under some cohomology restrictions, a versal deformation exists. Later, Fialowski and Fuchs, using this framework, gave a construction for a versal deformation.

Now, let us introduce some fundamental concepts and notations. The space of weighted densities of weight $\mu$ on $\mathbb{R}$ (or $\mu$-densities for short), denoted by:

$$
\mathcal{F}_{\mu}=\left\{f d x^{\mu}, f \in C^{\infty}\left(S^{1}\right)\right\}, \quad \mu \in \mathbb{R}
$$

is the space of sections of the line bundle $\left(T^{*} S^{1}\right)^{\otimes^{\mu}}$. The Lie algebra $\operatorname{Vect}\left(S^{1}\right)$ of vector fields $X_{h}=h \frac{d}{d x}$, where $h \in C^{\infty}\left(S^{1}\right)$, acts by the Lie derivative. Alternatively, this action can be written as follows:

$$
X_{g} \cdot\left(f d x^{\mu}\right)=L_{X_{g}}^{\mu}(f) d x^{\mu} \quad \text { with } L_{X_{g}}^{\mu}(f)=g f^{\prime}+\mu g^{\prime} f
$$

where $f^{\prime}, h^{\prime}$ are $\frac{d f}{d x}, \frac{d h}{d x}$.

We consider the Lie algebras $\mathfrak{s l}(2)$ and $\mathfrak{a f f}(1)$ which can be viewed as subalgebras of the Lie subalgebra of $\operatorname{Vect}\left(S^{1}\right)$ :

$$
\mathfrak{s l}(2)=\operatorname{Span}\left(X_{1}, X_{x}, X_{x^{2}}\right) \text { and } \mathfrak{a f f f}(1)=\operatorname{Span}\left(X_{1}, X_{x}\right) .
$$

Of course, $\mathfrak{a} \mathfrak{f} \mathfrak{f}(1)$ is a subalgebra of $\mathfrak{s l}(2)$. We also get families of infinite dimensional $\mathfrak{s l}(2)$-modules and $\mathfrak{a f f}(1)$-modules still denoted by $\mathcal{F}_{\lambda}$.

The study of multiparameter deformations of the standard embedding $\pi$ of the Lie algebra $\operatorname{Vect}\left(S^{1}\right)$ of smooth vector fields on $S^{1}$ into the Lie algebra of pseudodifferential symbols $\Psi \mathcal{D O}$ defined by $\pi\left(f(x) \partial_{x}\right)=f(x) \xi$ was carried out by Ovsienko and Roger in $[15,16]$. They have, essentially, studied polynomial deformations of the following form

$$
\pi(c)=\pi+\sum_{k \in \mathbb{Z}} \pi_{k}(c) \xi^{k}
$$

where $\pi_{k}(c): \operatorname{Vect}\left(S^{1}\right) \rightarrow C_{\mathbb{C}}^{\infty}\left(S^{1}\right)$ satisfy $\pi_{k}(0)=0$ and $\pi_{k} \equiv 0$ for $k$ sufficiently large.

In this paper, we are interested to the case of $\mathfrak{h}$-trivial deformations, where $\mathfrak{h}=\mathfrak{s l}(2)$ or $\mathfrak{a f f}(1)$. More precisely, we investigate the $\mathfrak{h}$-relative cohomology result by deducing the integrability condition for the infinitesimal deformation.

\section{Geometry of Space $S^{1}$}

\subsection{The space of tensor densities on $S^{1}$}

The Lie algebra, $\operatorname{Vect}\left(S^{1}\right)$, of vector fields on $S^{1}$ naturally acts, by the Lie derivative, on the space

$$
\mathcal{F}_{\lambda}=\left\{f d x^{\lambda}: f \in C^{\infty}\left(S^{1}\right)\right\}
$$


of weighted densities of degree $\lambda$. The Lie derivative $L_{X}^{\lambda}$ of the space $\mathcal{F}_{\lambda}$ along the vector field $X \frac{d}{d x}$ is defined by

$$
L_{X}^{\lambda}=X \partial_{x}+\lambda X^{\prime}
$$

where $X, f \in C^{\infty}\left(S^{1}\right)$ and $X^{\prime}:=\frac{d X}{d x}$. More precisely, for all $f d x^{\lambda} \in \mathcal{F}_{\lambda}$ we have

$$
L_{X}^{\lambda}\left(f d x^{\lambda}\right)=\left(X f^{\prime}+\lambda f X^{\prime}\right) d x^{\lambda} .
$$

\subsection{The standard embedding}

The Lie algebra $\operatorname{Vect}\left(S^{1}\right)$ of vector fields on a manifold $S^{1}$ has a natural embedding into the Poisson Lie algebra of functions on $T^{*} S^{1}$. It is defined by the standard action of the Lie algebra of vector fields on the cotangent bundle, the explicit formula is

$$
\pi\left(X_{f}\right)=f \xi
$$

The main purpose of this paper is to study $\mathfrak{a} \mathfrak{f}(1)$-trivial deformations of the standard embedding (2.2).

\subsection{The Poisson Lie algebra of formal symbols}

\subsubsection{Deformations inside $C^{\infty}\left(T^{*} S^{1}\right)$}

Consider the Poisson Lie algebra of smooth functions on $T^{*} S^{1}$. In this case, the problem of deformation of the embedding (2.2) has an elementary solution. The $\operatorname{Vect}\left(S^{1}\right)$ embedding (2.2) into $C^{\infty}\left(T^{*} S^{1}\right)$ has the unique (well-known) nontrivial deformation. Indeed, given an arbitrary volume form on $S^{1}$, the expression:

$$
\pi_{\lambda}(X)=X \xi+\lambda \operatorname{div} X,
$$

where $\lambda \in \mathbb{R}$ defines an embedding of $\operatorname{Vect}\left(S^{1}\right)$ into $C^{\infty}\left(T^{*} S^{1}\right)$. The linear map: $X \rightarrow \operatorname{div} X$ is the unique nontrivial 1-cocycle on $\operatorname{Vect}\left(S^{1}\right)$ with values $C^{\infty}\left(S^{1}\right) \subset$ $C^{\infty}\left(T^{*} S^{1}\right)$.

\subsubsection{Definition: Two Poisson Lie algebras of formal symbols}

Let us consider the following Poisson Lie algebra on the cotangent bundle with zero section removed, $\dot{T}^{*} S^{1}=T^{*} S^{1} \backslash S^{1}$ :

(i) The Lie algebra $P$ of functions on $\dot{T}^{*} S^{1}$.

(ii) The Lie algebra $\mathcal{P}$ of formal Laurent series on $\dot{T}^{*} S^{1}$.

Lie algebras $P$ and $\mathcal{P}$ can be interpreted as classical limits of the algebra of formal symbols of pseudodifferential operators on $S^{1}$. In both cases, the Poisson bracket is defined by the usual formula:

$$
\{F, G\}=\frac{\partial F}{\partial \xi} \frac{\partial G}{\partial x}-\frac{\partial F}{\partial x} \frac{\partial G}{\partial \xi} .
$$


As vector spaces, Lie algebras $P$ and $\mathcal{P}$ have the following form:

$$
\left.P=C^{\infty}\left(S^{1}\right) \otimes \mathbb{C}\left[\xi, \xi^{-1}\right] \text { and } \mathcal{P}=C^{\infty}\left(S^{1}\right) \otimes \mathbb{C}\left[\xi, \xi^{-1}\right]\right],
$$

where $\left.\mathbb{C}\left[\xi, \xi^{-1}\right]\right]$ is the space of Laurent series in one formal indeterminate. Elements of both algebras $P$ and $\mathcal{P}$ can be written in the following form:

$$
F\left(x, \xi, \xi^{-1}\right)=\sum_{k \in \mathbb{Z}} \xi^{k} f_{k}(x),
$$

where the coefficients $f_{k}(x)$ are periodic functions: $f_{k}(x+2 \pi)=f_{k}(x)$. In the case of algebra $P$, one supposes that the coefficients $f_{k} \equiv 0$, if $|k|$ is sufficiently large; for $\mathcal{P}$ the condition is: $f_{k} \equiv 0$, if $k$ is sufficiently large.

Lemma 2.1 ([16]). The Lie algebra $\mathcal{P}$ has the following decomposition as a $\operatorname{Vect}\left(S^{1}\right)$-module.

$$
\mathcal{P}=\oplus_{m \geq 0} \mathcal{F}_{m} \oplus \prod_{m<0} \mathcal{F}_{m}
$$

Theorem 2.2 (Ovsienko-Roger [16]). The space $\mathrm{H}^{1}\left(\operatorname{Vect}\left(S^{1}\right), \mathcal{P}\right)$ has the following structure:

$$
\mathrm{H}^{1}\left(\operatorname{Vect}\left(S^{1}\right), \mathcal{P}\right)=\mathbb{R}^{4} .
$$

The nontrivial cocycles generating the cohomology groups are as follows:

$$
\left\{\begin{array}{l}
c_{0}(f \partial)=f \\
C_{0}(f \partial)=f^{\prime} \\
C_{1}(f \partial)=f^{\prime \prime} \xi^{-1} \\
C_{2}(f \partial)=f^{\prime \prime \prime} \xi^{-2} .
\end{array}\right.
$$

\subsection{Pseudodifferential operators on $S^{1}$}

To every pseudodifferential operator $F$ on $S^{1}$, one associates its symbol $[6,16]$. Let $\Psi \mathcal{D O}$ be the space of pseudodifferential symbols on $S^{1} \quad[15,16]$. The order of $\Psi \mathcal{D O}$ is defined to be

$$
\operatorname{ord}(F)=\left\{\sup k \in \mathbb{Z} \mid f_{k}(x) \neq 0\right\} \quad \text { for any } \quad F\left(x, \xi, \xi^{-1}\right)=\sum_{k \in \mathbb{Z}} \xi^{k} f_{k}(x) \in \Psi \mathcal{D O},
$$

where $f_{k}(x) \in C^{\infty}(\mathbb{R})$ with $f_{k}=0$ for $k$ sufficiently large. By means of the two natural derivations on $\Psi \mathcal{D O}$

$$
\partial_{\xi}: \sum_{k} f_{k} \xi^{k} \mapsto \sum_{k} k f_{k} \xi^{k-1} \quad \text { and } \quad \partial_{x}: \sum_{k} f_{k} \xi^{k} \mapsto \sum_{k} f_{k}^{\prime} \xi^{k}
$$

one defines a natural Poisson bracket:

$$
\{F, G\}=\frac{\partial F}{\partial \xi} \frac{\partial G}{\partial x}-\frac{\partial F}{\partial x} \frac{\partial G}{\partial \xi} \quad \text { for any } \quad F, G \in \Psi \mathcal{D O} \text {. }
$$


On the space of pseudodifferential symbols an associative algebra structure is defined by the rule [15]

$$
F \circ G=\sum_{k \geq 0} \frac{1}{k !}: \frac{\partial^{k} F}{\partial \xi^{k}} \frac{\partial^{k} G}{\partial x^{k}}:,
$$

where $: \cdot$ : stands for the "normal ordering" defined as

$$
: f(x) \xi^{k} g(x) \xi^{\ell}:=f(x) g(x) \xi^{k+\ell} .
$$

This is a natural generalization of the Wick product. As usual, we can also define the Lie bracket associated with the o-product $[F, G]=F \circ G-G \circ F$; we denote the Lie algebra with this structure by $\Psi \mathcal{D O} \mathcal{O}_{L}$ to distinguish from the Poisson Lie algebra structure.

Consider the family of associative laws on $\Psi \mathcal{D O}$ depending on a parameter $h \in] 0,1]$ :

$$
A \circ_{h} B=\sum_{n \geq 0,} \frac{h^{n}}{n !}\left(\partial_{\xi}^{n} A\right)\left(\partial_{x}^{n} B\right) .
$$

Denote by $\Psi \mathcal{D O} \mathcal{O}_{h}$ the associative algebra of pseudodifferential operators on $S^{1}$ equipped with the multiplication $\circ_{h}$. It is clear that all the associative algebras $\Psi \mathcal{D O} \mathcal{O}_{h}$ are isomorphic to each other.

For the commutator $[A, B]_{h}:=A \circ_{h} B-B \circ_{h} A$, one has

$$
[A, B]_{h}=\{A, B\}+O(h)
$$

and therefore $\lim _{h \rightarrow 0}[A, B]_{h}=\{A, B\}$, where we identify $\mathcal{P}$ with $\Psi \mathcal{D O}$ as vector spaces. Hence, the Lie algebra $\Psi \mathcal{D O}$ contracts to the Poisson Lie algebra $\mathcal{P}$.

Theorem 2.3 (Ovsienko-Roger [15]). The space $\mathrm{H}^{1}\left(\operatorname{Vect}\left(S^{1}\right), \Psi \mathcal{D O}\right)$ has the following structure:

$$
\mathrm{H}^{1}\left(\operatorname{Vect}\left(S^{1}\right), \Psi \mathcal{D O}\right)=\mathbb{R}^{4} .
$$

The nontrivial cocycles generating the cohomology groups are as follows:

$$
\left\{\begin{array}{l}
\Theta_{0}(f \partial)=f \\
\Theta_{1}(f \partial)=f^{\prime} \\
\Theta_{2}(f \partial)=\sum_{n=2}^{\infty}(-1)^{n-1} \frac{2(n-3)}{n} f^{(n)} \xi^{-n+1} \\
\Theta_{3}(f \partial)=\sum_{n=2}^{\infty}(-1)^{n} \frac{3(n-1)}{n+1} f^{(n+1)} \xi^{-n}
\end{array}\right.
$$

In $[15,16]$, Ovsienko and Roger classify nontrivial deformations of the standard embedding of the Lie algebra $\operatorname{Vect}\left(S^{1}\right)$ the Lie algebra of functions on the cotangent bundle $T^{*} S^{1}$ with respect to the Poisson bracket and into the Lie algebra $\Psi \mathcal{D O}$ of pseudodifferential symbols on $S^{1}$. Now, let $\mathfrak{h}$ be Lie subagebra of $\operatorname{Vect}\left(S^{1}\right)$, we classify all the $\mathfrak{h}$-trivial deformations of the standard embedding. 


\section{Statement of the Problem}

\section{1. h-trivial deformation of the $\operatorname{Vect}\left(S^{1}\right)$ inside $P$}

We classify all the $\mathfrak{h}$-trivial deformations of the standard embedding (2.2) of $\operatorname{Vect}\left(S^{1}\right)$ into $P$. That means we study linear $\mathfrak{h}$-trivial maps $\pi^{c}: \operatorname{Vect}\left(S^{1}\right) \rightarrow P[[c]]$ to the Lie algebra of series in a formal parameter in $c$. Such a map has the following form:

$$
\pi^{c}=\pi+c \pi_{1}+c_{2} \pi_{2}+\cdots,
$$

where $\pi_{k}: \operatorname{Vect}\left(S^{1}\right) \rightarrow P$ are linear $\mathfrak{h}$-trivial maps, such that formal homomorphism condition is satisfied.

\section{2. h-trivial deformation of the $\operatorname{Vect}\left(S^{1}\right)$ inside $\Psi \mathcal{D O}$}

The main purpose of this paper is to study $\mathfrak{h}$-trivial deformations of the canonical embedding

$$
\begin{aligned}
\rho_{0}: \operatorname{Vect}\left(S^{1}\right) & \rightarrow \Psi \mathcal{D O} \\
\rho_{0}\left(X_{F}\right) & =F \xi .
\end{aligned}
$$

\section{3. h-trivial deformation of the $\operatorname{Vect}\left(S^{1}\right)$ inside $\mathcal{P}$}

We classify all the $\mathfrak{h}$-trivial deformations of the standard embedding (2.2) of $\operatorname{Vect}\left(S^{1}\right)$ into $\mathcal{P}$. In other words, we consider homomorphisms of the following form:

$$
\pi=\pi+\sum_{k \in \mathbb{Z}} \pi_{k}(c) \xi^{k}
$$

where $c=c_{1}, \ldots, c_{n} \in \mathbb{R}$ (or $\mathbb{C}$ ) are parameters of deformation, each linear map $\pi_{k}(c): \operatorname{Vect}\left(S^{1}\right) \rightarrow C^{\infty}\left(S^{1}\right)$ being polynomial in $c, \pi_{k}(0)=0$ and $\pi_{k} \equiv 0$ if $k>0$ is sufficiently large.

\subsubsection{Deformation theory}

Deformation theory of Lie algebra homomorphisms was first considered with one parameter $[10,14]$. Recently, multiparameter deformations of Lie algebras and their modules were intensively studied $[1,2,4,5,8,9,15,16]$. Recall the basics of this theory.

Let $\rho_{0}: \operatorname{Vect}\left(S^{1}\right) \rightarrow \Psi \mathcal{D O}$ be an embedding of Lie algebras. A formal $\mathfrak{h}$-trivial deformation of $\rho_{0}$ is a formal series

$$
\rho(t)=\rho_{0}+\sum_{i=1}^{m} t_{i} \rho_{i}+\sum_{i, j=1}^{m} t_{i} t_{j} \rho_{i j}^{(2)}+\cdots,
$$


where the $\rho_{i}, \rho_{i j}^{(2)}, \ldots$, are linear maps from $\operatorname{Vect}\left(S^{1}\right)$ to $\Psi \mathcal{D O}$ with $t=$ $\left(t_{1}, \ldots, t_{m}\right) \in \mathbb{C} \llbracket t_{1}, \ldots, t_{m} \rrbracket$, and so on, such that the map

$$
\rho(t): \operatorname{Vect}\left(S^{1}\right) \rightarrow \mathbb{C} \llbracket t_{1}, \ldots, t_{m} \rrbracket \otimes \Psi \mathcal{D O}
$$

satisfies the homomorphism condition in any order in $t=\left(t_{1}, \ldots, t_{m}\right)$.

Two formal $\mathfrak{h}$-trivial deformations $\rho$ and $\widetilde{\rho}$ are said to be equivalent if $\widetilde{\rho}=I_{t} \circ \rho$ for an inner automorphism

$$
I_{t}: \mathbb{C} \llbracket t \rrbracket \otimes \Psi \mathcal{D O} \rightarrow \mathbb{C} \llbracket t \rrbracket \otimes \Psi \mathcal{D O}
$$

of the form

$$
\begin{array}{r}
I_{t}=\exp \left(\sum_{i=1}^{m} t_{i} \operatorname{ad}_{F_{i}}+\sum_{i, j} t_{i} t_{j} \operatorname{ad}_{F_{i, j}}+\cdots\right) \text { for some } \\
F_{i}, F_{i, j}, \ldots, F_{i_{1} \ldots i_{k}} \in \Psi \mathcal{D O} .
\end{array}
$$

In many cases, we have to consider polynomial $\mathfrak{h}$-trivial deformations instead of formal ones: the formal series can sometimes be cut off, and the formal parameter be replaced by complex coefficients. More precisely, according to $[15,16]$, a polynomial $\mathfrak{h}$-trivial deformation of the standard embedding (3.1) is a Lie algebra homomorphism $\rho(c): \operatorname{Vect}\left(S^{1}\right) \rightarrow \Psi \mathcal{D O}$ of the following form:

$$
\rho(c)=\rho_{0}+\sum_{k \in \mathbb{Z}} \rho_{k}(c),
$$

where $\left.\rho_{k}(c): \operatorname{Vect}\left(S^{1}\right)\right) \rightarrow \mathcal{P}_{k}$ are linear maps with $\mathcal{P}_{k}$ as in (2.4), polynomial in parameter $c=\left(c_{1}, \ldots, c_{m}\right) \in \mathbb{C}^{m}$ and such that $\rho_{k} \equiv 0$ for $k$ sufficiently large.

It is reported that theory of polynomial $\mathfrak{h}$-trivial deformations seems to be richer than that of formal ones. The equivalence problem for polynomial $\mathfrak{h}$-trivial deformations has additional interesting aspects related to parameter transformations.

\subsubsection{Infinitesimal $\mathfrak{h}$-trivial deformations and the first relative cohomology}

Deformations (3.2) and (3.5), modulo second-order terms in $t$ and $c$, respectively, are called infinitesimal. Infinitesimal $\mathfrak{h}$-trivial deformations of a Lie algebra homomorphism $\rho$ from $\mathfrak{g}$ into $\mathfrak{b}$ are classified by the first cohomology $\mathrm{H}^{1}(\mathfrak{g}, \mathfrak{h}, \mathfrak{b})$, where $\mathfrak{b}$ is a $\mathfrak{g}$-module through $\rho$ and $\mathfrak{h}$ is subalgebra of $\mathfrak{g}$. Namely, the first-order terms $\rho_{i}$ in (3.2) and $\left.\frac{\partial \rho(c)}{\partial c_{i}}\right|_{c=0}$ in (3.5) are 1-cocycles. Two infinitesimal $\mathfrak{h}$-trivial deformations are said to be equivalent if the corresponding cocycles are cohomologous. Conversely, given a Lie algebra homomorphism $\rho: \mathfrak{g} \rightarrow \mathfrak{b}$, an arbitrary 1-cocycle $\rho_{1} \in Z^{1}(\mathfrak{g}, \mathfrak{h}, \mathfrak{b})$ defines an infinitesimal deformations of $\rho$.

The obstructions for existence of a $\mathfrak{h}$-trivial deformation (3.2) lie in $\mathrm{H}^{2}\left(\operatorname{Vect}\left(S^{1}\right), \mathfrak{h}, \Psi \mathcal{D} \mathcal{O}\right)$. Indeed, setting

$$
\varphi_{t}=\rho-\rho_{0}, \quad \rho^{(1)}=\sum t_{i} \rho_{i}, \quad \rho^{(2)}=\sum t_{i} t_{j} \rho_{i j}^{(2)}, \ldots,
$$


we can rewrite the relation (3.2) in the following way:

$$
\left[\varphi_{t}(x), \rho_{0}(y)\right]+\left[\rho_{0}(x), \varphi_{t}(y)\right]-\varphi_{t}([x, y])+\sum_{i, j>0}\left[\rho^{(i)}(x), \rho^{(j)}(y)\right]=0 .
$$

The first three terms are $\left(\delta \varphi_{t}\right)(x, y)$, where $\delta$ stands for the coboundary. For arbitrary linear maps $\gamma_{1}, \gamma_{2}: \mathfrak{g} \rightarrow \mathfrak{b}$, consider the standard cup-product: $\llbracket \gamma_{1}, \gamma_{2} \rrbracket$ : $\mathfrak{g} \otimes \mathfrak{g} \rightarrow \mathfrak{b}$ defined by:

$$
\llbracket \gamma_{1}, \gamma_{2} \rrbracket(x, y)=\left[\gamma_{1}(x), \gamma_{2}(y)\right]+\left[\gamma_{2}(x), \gamma_{1}(y)\right] .
$$

The relation (3.6) becomes now equivalent to

$$
\delta \varphi_{t}=-\frac{1}{2} \llbracket \varphi_{t}, \varphi_{t} \rrbracket .
$$

Expanding (3.8) in power series in $t$, we obtain the following equation for $\rho^{(k)}$ :

$$
\delta \rho^{(k)}=-\frac{1}{2} \sum_{\substack{i+j=k \\ i, j>0}} \llbracket \rho^{(i)}, \rho^{(j)} \rrbracket .
$$

The right-hand side of (3.9) is a 2-cocycle whose coefficients are homogeneous of degree $k$ polynomials in $t$, so the cohomology class of this cocycle is an obstruction for existence of the solutions. The first nontrivial relation $\delta \rho^{(2)}=-\frac{1}{2} \llbracket \rho^{(1)}, \rho^{(1)} \rrbracket$ gives the first obstruction to integration of an infinitesimal $\mathfrak{h}$-trivial deformation. Thus, considering the coefficient of $t_{i} t_{j}$, we get

$$
\delta \rho_{i, j}^{(2)}=-\frac{1}{2} \llbracket \rho_{i}, \rho_{j} \rrbracket .
$$

This relation is precisely the condition for the 2 -cocycle $\llbracket \rho_{i}, \rho_{j} \rrbracket$ to be a coboundary.

\section{4. aff(1)-Trivial Deformation of the $\operatorname{Vect}\left(S^{1}\right)$ Inside $P$}

\section{1. $\mathfrak{a f f}(1)$-relative cohomology acting on $P$}

\section{Corollary 4.1.}

$$
\mathrm{H}^{1}\left(\operatorname{Vect}\left(S^{1}\right), \mathfrak{a} \mathfrak{f} \mathfrak{f}(1), P\right)=\mathrm{H}^{1}\left(\operatorname{Vect}\left(S^{1}\right), \mathfrak{a} \mathfrak{f} \mathfrak{f}(1), \mathcal{P}\right)=\mathbb{R}^{2} .
$$

The nontrivial cocycles generating the relative cohomology groups are as follows:

$$
\left\{\begin{array}{l}
C_{1}(f \partial)=f^{\prime \prime} \xi^{-1} \\
C_{2}(f \partial)=f^{\prime \prime \prime} \xi^{-2}
\end{array}\right.
$$

\subsection{Second-order integrability conditions}

Proposition 4.2. Every infinitesimal afff(1)-trivial deformation of the standard embedding of $\operatorname{Vect}\left(S^{1}\right)$ into $P$ is equivalent to:

$$
f \partial \mapsto f \xi+c_{1} f^{\prime \prime} \xi^{-1}+c_{2} f^{\prime \prime \prime} \xi^{-2},
$$

where $c_{1}, c_{2} \in \mathbb{R}($ or $\mathbb{C})$ are parameters. 
In this section, we obtain the integrability conditions for the infinitesimal deformation (4.2). Assume that the infinitesimal deformation (4.2) can be integrated to a formal deformation

$$
\varrho_{X}=\rho_{X}+\rho_{X}^{(1)}+\rho_{X}^{(2)}+\rho_{X}^{(3)}+\cdots,
$$

where $\rho_{X}^{(1)}$ is given by (4.1) and $\rho_{X}^{(2)}$ is a quadratic polynomial in $c_{i}$ whose coefficients are elements of $P$ vanishing on $\mathfrak{a f f}(1)$. We compute the conditions for the secondorder terms $\rho^{(2)}$. The homomorphism condition

$$
\left[\varrho_{X}, \varrho_{Y}\right]=\varrho_{[X, Y]}
$$

gives for the second-order terms the following (Maurer-Cartan) equation

$$
\partial \rho^{(2)}=-\frac{1}{2} \llbracket \rho^{(1)}, \rho^{(1)} \rrbracket .
$$

The right-hand side of (4.4) is a cup-product of aff(1)-relative 1-cocycles, so it is automatically a afff(1)-relative 2-cocycle. More precisely, Eq. (4.4) can be expressed as follows:

$$
\partial \rho^{(2)}=-\frac{1}{2}\left[\left[\sum_{i=1}^{2} c_{i} C_{i}, \sum_{i=1}^{2} c_{i} C_{i}\right]\right]
$$

therefore, let us consider the $\mathfrak{a f f}(1)$-relative 2-cocycles $\Omega_{i, j} \in Z_{\text {diff }}^{2}\left(\operatorname{Vect}\left(S^{1}\right), \mathfrak{a} \mathfrak{f} \mathfrak{f}(1)\right.$, $P)$, defined by

$$
\Omega_{i, j}=\llbracket C_{i}, C_{j} \rrbracket .
$$

It is easy to see that $\Omega_{i, i}=0$, for $i=1,2$. The second-order integrability conditions are determined by the fact that any map 2-cocycles $\Omega_{i, j}$, for $i \neq j, i, j \in\{1,2\}$, must be a $\mathfrak{a f f}(1)$-relative 2 -coboundary. More precisely, $\Omega_{i, j}$ must coincide, up to a scalar factor, with $\partial b$, where $b: \operatorname{Vect}\left(S^{1}\right) \rightarrow P, \mathfrak{a f f}(1)$-invariant. We need the following lemma.

Lemma 4.3. Let $b \in C^{1}\left(\operatorname{Vect}\left(S^{1}\right), \mathfrak{a f f}(1), P\right)$ defined as follows: for $X \frac{d}{d x} \in$ $\operatorname{Vect}\left(S^{1}\right)$

$$
b\left(X_{f}\right)=\sum_{j=2}^{9} \alpha_{j} f^{(j)} \xi^{(9-j)},
$$

where the coefficients $\alpha_{j}$ are constant. Then the map $\partial b: \operatorname{Vect}\left(S^{1}\right) \times \operatorname{Vect}\left(S^{1}\right) \rightarrow P$ is given by

$$
\begin{aligned}
\partial b(X, Y)(f)= & \alpha_{6}\left(2\left(f^{\prime} g^{(6)}-f^{(6)} g^{\prime}\right)-9\left(f^{(2)} g^{(5)}-f^{(5)} g^{(2)}\right)\right. \\
& \left.\left.\left.+5\left(f^{(3)} g^{(4)}-f^{(4)}\right) g^{(3)}\right)\right) \xi^{-3} 5 \alpha_{5}\left(f^{(2)} g^{(4)}-f^{(4)}\right) g^{(2)}\right) \xi^{-4} .
\end{aligned}
$$

Proof. Straightforward computation. 
We split these conditions into two families which we explain in the two following propositions. Let us first consider the following functions in $c$, where $c$ is the family of parameters $\left(c_{i}\right)$

$$
\begin{aligned}
& \omega_{i, i}(c)=c_{i}^{2}, \quad \text { if } i \in\{1,2\}, \\
& \omega_{1,2}(c)=c_{1} c_{2} .
\end{aligned}
$$

These functions $\omega_{i, j}(c), i, j=1,2$, will appear as coefficients for some maps in $P$ and they will be used in the expressions of integrability conditions. More precisely, we will show that the second term $\rho^{(2)}$ is of the form $\rho^{(2)}=\sum \omega_{i, j}(c) b_{i j}$.

\section{Proposition 4.4.}

(1) For $i \in\{1,2\}, \Omega_{i, i}(f \partial, g \partial)=0$.

(2) The map $\Omega_{1,2}(f \partial, g \partial)=\omega_{1,2}\left(f^{(4)} g^{(2)}-f^{(2)} g^{(4)}\right) \xi^{-4}$, is a trivial $\mathfrak{a f f}(1)$-relative 2-cocycle.

Proof. (1) For $i \in\{1,2\}$, we have

$$
\Omega_{i, i}=c_{i}^{2} \llbracket C_{i}, C_{i} \rrbracket .
$$

By a direct computation, we show that

$$
\llbracket C_{i}, C_{i} \rrbracket(f \partial, g \partial)=\left\{C_{i}, C_{i}\right\}(f \partial, g \partial)+\left\{C_{i}, C_{i}\right\}(f \partial, g \partial)=0 .
$$

(2) Note that hereafter, some singular values of the parameter $i \neq j$ appear because the $\mathfrak{a f f}(1)$-relative 2-cocycles $\llbracket C_{i}, C_{j} \rrbracket(f \partial, g \partial)$ exist only for $i \neq j$.

(i) By a direct computation, we show that

$$
\begin{aligned}
\llbracket C_{1}, C_{2} \rrbracket(f \partial, g \partial) & =\left\{C_{1}, C_{2}\right\}(f \partial, g \partial)+\left\{C_{2}, C_{1}\right\}(f \partial, g \partial) \\
& =\omega_{1,2}\left(f^{(4)} g^{(2)}-f^{(2)} g^{(4)}\right) \xi^{-4} .
\end{aligned}
$$

(ii) We exhibit some reals $\alpha$ and $\beta$ such that

$$
\alpha \Omega_{1,2}=\beta \partial b .
$$

By a direct computation, we show that

$$
\Omega_{1,2}=\omega_{1,2}(c) \partial b .
$$

Hence, there are no conditions on $\Omega_{1,2}$.

Our main result in this section is the following theorem.

Theorem 4.5. There are no integrability conditions for second-order integrability of the aff(1)-trivial infinitesimal deformation (4.2).

Proof. Of course, these conditions are necessary as was shown in Proposition 4.1. Now, under these conditions, the second term $\rho^{(2)}$ of the $\mathfrak{a f f}(1)$-trivial infinitesimal deformation (4.2) is a solution of the Maurer-Cartan equation (4.5). This solution is 
defined up to a 1-coboundary and it has been shown in $[10,1]$ that different choices of solutions of the Maurer-Cartan equation correspond to equivalent deformations. Thus, we can always choose

$$
\rho^{(2)}=\frac{1}{2} \omega_{1,2}(c) b\left(X_{f}\right)=\frac{1}{2} \omega_{1,2}(c) f^{(5)} \xi^{-4} .
$$

Theorem 4.5 is proved.

\subsection{Third and fourth-order integrability conditions}

\subsubsection{Computing the third-order Maurer-Cartan equation}

Now, we reconsider the formal deformation (4.3) which is a formal power series in the parameters $c_{i}$ with coefficients in $P$. We suppose that the second-order integrability conditions are satisfied. So, the third-order terms of (4.3) are solutions of the (Maurer-Cartan) equation

$$
\partial \rho^{(3)}=-\frac{1}{2} \sum_{i+j=3} \llbracket \rho^{(i)}, \rho^{(j)} \rrbracket .
$$

As in the previous section, we can write

$$
\partial \rho^{(3)}=-\frac{1}{2} \sum_{i=1}^{2} \Xi_{i},
$$

where $\Xi_{i}$ are maps from $\operatorname{Vect}\left(S^{1}\right)^{\otimes 2}$ to $P$. The third-order term $\rho^{(3)}$ of the $\mathfrak{a} \mathfrak{f} f(1)$ trivial formal deformation (4.3) is a solution of (4.10). So, the 2-cochains $\Xi_{i}$ must satisfy $\Xi_{i}=\partial b$ and then the third-order integrability conditions are deduced from this fact.

We compute successively the $\Xi_{i}$ for $1=1,2$ and we resolve $\Xi_{i}=\partial b$ to get the corresponding third-order integrability conditions.

Here, we mention that the maps $\Xi_{i}$ are 2-cochains, but they are not necessarily 2-cocycles because they are not cup-products of 1-cocycles like the maps $\Omega_{i, j}$. Indeed, $\rho^{(2)}$ is not necessarily a 1-cocycle.

\subsubsection{Third-order integrability conditions}

Proposition 4.6. We have the following third-order integrability conditions of the infinitesimal deformation (4.2),

$$
\begin{aligned}
& c_{1} \omega_{1,2}(c)=c_{1}^{2} c_{2}=0, \\
& c_{2} \omega_{1,2}(c)=c_{1} c_{2}^{2}=0 .
\end{aligned}
$$

We need the following lemmas. 
Lemma 4.7. Let $b_{6} \in C^{1}\left(\operatorname{Vect}\left(S^{1}\right), \mathfrak{a f f}(1), P\right)$ defined as follows: for $f \frac{d}{d x} \in$ $\operatorname{Vect}\left(S^{1}\right)$

$$
b_{6}\left(X_{f}\right)=\alpha_{8,5} f^{(8)} \xi^{-5}+\alpha_{7,6} f^{(7)} \xi^{-6},
$$

where the coefficients $\alpha_{7,6}$ and $\alpha_{8,5}$ are constant. Then the map $\partial b_{6}: \operatorname{Vect}\left(S^{1}\right)^{\otimes 2} \rightarrow$ $P$ is given by

$$
\begin{aligned}
\partial b_{6}\left(X_{f}, X_{g}\right)(f)= & 2 \alpha_{8,5}\left(\left(f^{(8)} g^{\prime}-f^{\prime} g^{8}\right)+10\left(f^{(7)} g^{(2)}-f^{(2)} g^{(7)}\right)\right) \xi^{-5} \\
& \left.+14 \alpha_{8,5}\left(2\left(f^{(6)} g^{(3)}-f^{(3)}\right) g^{(6)}\right)+\left(f^{(5)} g^{(4)}-f^{(4)} g^{(5)}\right)\right) \xi^{-5} \\
& +14 \alpha_{7,6}\left(\left(f^{(6)} g^{(2)}-f^{(2)} g^{(6)}\right)+\left(f^{(5)} g^{(3)}-f^{(3)} g^{(5)}\right)\right) \xi^{-6}
\end{aligned}
$$

Proof. Straightforward computation.

Lemma 4.8. Let $b_{7} \in C^{1}\left(\operatorname{Vect}\left(S^{1}\right), \mathfrak{a f f}(1), P\right)$ defined as follows: for $f \frac{d}{d x} \in$ $\operatorname{Vect}\left(S^{1}\right)$

$$
b_{7}\left(X_{f}\right)=\alpha_{9,6} f^{(9)} \xi^{-6}+\alpha_{8,7} f^{(8)} \xi^{-7}
$$

where the coefficients $\alpha_{9,6}$ and $\alpha_{8,7}$ are constant.

Then $\partial b_{7} \in C^{2}\left(\operatorname{Vect}\left(S^{1}\right), \mathfrak{a f f}(1), P\right)$ has the general following form: for $f \frac{d}{d x}, g \frac{d}{d x} \in \operatorname{Vect}\left(S^{1}\right)$

$$
\begin{aligned}
\partial b_{7}\left(X_{f}, Y_{g}\right)(f)= & 2 \alpha_{9,6}\left(f^{(9)} g^{\prime}-f^{\prime} g^{9}\right) \xi^{-6}+27 \alpha_{9,6}\left(f^{(8)} g^{(2)}-f^{(2)} g^{(8)}\right) \xi^{-6} \\
& +38 \alpha_{9,6}\left(f^{(7)} g^{(3)}-f^{(3)}\right) g^{(7)} \xi^{-6}+42 \alpha_{9,6}\left(f^{(6)} g^{(4)}-f^{(4)} g^{(6)}\right) \xi^{-6} \\
& +20 \alpha_{8,7}\left(f^{(7)} g^{(2)}-f^{(2)} g^{(7)}\right) \xi^{-7}+28 \alpha_{8,7}\left(f^{(6)} g^{(3)}-f^{(3)} g^{(6)}\right) \xi^{-7} \\
& +14 \alpha_{8,7}\left(f^{(5)} g^{(4)}-f^{(4)} g^{(5)}\right) \xi^{-7} .
\end{aligned}
$$

Proof. We have

$$
\Xi_{1}=c_{1} \omega_{1,2}(c) \llbracket C_{1}, b \rrbracket
$$

and

$$
\Xi_{2}=c_{2} \omega_{1,2}(c) \llbracket C_{2}, b \rrbracket .
$$

By a direct computation, we show that the 2-cochains

$$
\Xi_{1}=c_{1}^{2} c_{2}\left(\left(f^{(6)} g^{(2)}-f^{(2)} g^{(6)}\right)+4\left(f^{(3)} g^{(5)}-f^{(5)} g^{(3)}\right)\right) \xi^{-6} \quad \text { and } \quad \partial b_{6}
$$

are linearly independent. In the same manner, $\Xi_{2}=c_{1} c_{2}^{2}\left(\left(f^{(6)} g^{(3)}-f^{(3)} g^{(6)}\right)+\right.$ $\left.4\left(f^{(4)} g^{(5)}-f^{(5)} g^{(4)}\right)\right) \xi^{-7}$ and $\partial b_{7}$ are linearly independent. Thus,

$$
\begin{gathered}
c_{1}^{2} c_{2}=0, \\
c_{1} c_{2}^{2}=0 .
\end{gathered}
$$

We compute these 2-cochains and then we check the corresponding integrability conditions. 


\subsubsection{Fourth-order integrability conditions}

Proposition 4.9. There are no integrability conditions for the fourth-order integrability conditions of the infinitesimal deformation (4.2).

Proof. These conditions come from the fact that the fourth term $\rho^{(4)}$ must satisfy:

$$
\partial \rho^{(4)}=-\frac{1}{2} \llbracket \rho^{(2)}, \rho^{(2)} \rrbracket .
$$

Indeed, we can always reduce $\rho^{(3)}$ to zero by equivalence.

The following theorem is our main result.

Theorem 4.10. The third-order conditions (4.11) are necessary and sufficient for the complete integrability of the infinitesimal deformation (4.2). Moreover, any formal afff(1)-trivial deformation of the Lie derivative $\rho_{X}$ on the space of symbols $P$ is equivalent to a polynomial one of degree equal or less than 2.

Proof. Clearly, all these conditions are necessary. So, let us prove that they are also sufficient. As in the proof of Theorem 4.5, the solution $\rho^{(3)}$ of the MaurerCartan equation (4.9) is defined up to a 1-coboundary, thus, we can always reduce $\rho^{(3)}$ to zero by equivalence. Moreover, by recurrence, the highest-order terms $\rho^{(m)}$ satisfy the equation $\partial \rho^{(m)}=0$ and can also be reduced to the identically zero map. This completes the proof of Theorem 4.10.

\section{5. $\mathfrak{a f f}(1)$-Trivial Deformation of the $\operatorname{Vect}\left(S^{1}\right)$ Inside $\Psi \mathcal{D O}$}

\section{1. aff(1)-relative cohomology acting on $\Psi \mathcal{D O}$}

\section{Corollary 5.1.}

$$
\mathrm{H}^{1}\left(\operatorname{Vect}\left(S^{1}\right), \mathfrak{a f f}(1), \Psi \mathcal{D O}\right)=\mathbb{R}^{2} .
$$

The nontrivial cocycles generating the relative cohomology groups are as follows:

$$
\left\{\begin{array}{l}
\Theta_{2}(f \partial)=\sum_{n=2}^{\infty}(-1)^{n-1} \frac{2(n-3)}{n} f^{(n)} \xi^{-n+1} \\
\Theta_{3}(f \partial)=\sum_{n=2}^{\infty}(-1)^{n} \frac{3(n-1)}{n+1} f^{(n+1)} \xi^{-n}
\end{array}\right.
$$

\subsection{Infinitesimal deformations}

Proposition 5.2. Every infinitesimal afff(1)-trivial deformation of the standard embedding of $\operatorname{Vect}\left(S^{1}\right)$ into $\Psi \mathcal{D O}$ is equivalent to

$$
\tilde{\pi}: f \partial \mapsto f \xi+c_{2} \Theta_{2}(f \partial)+c_{3} \Theta_{3}(f \partial),
$$

where $c_{2}, c_{3} \in \mathbb{R}($ or $\mathbb{C})$ are parameters. 


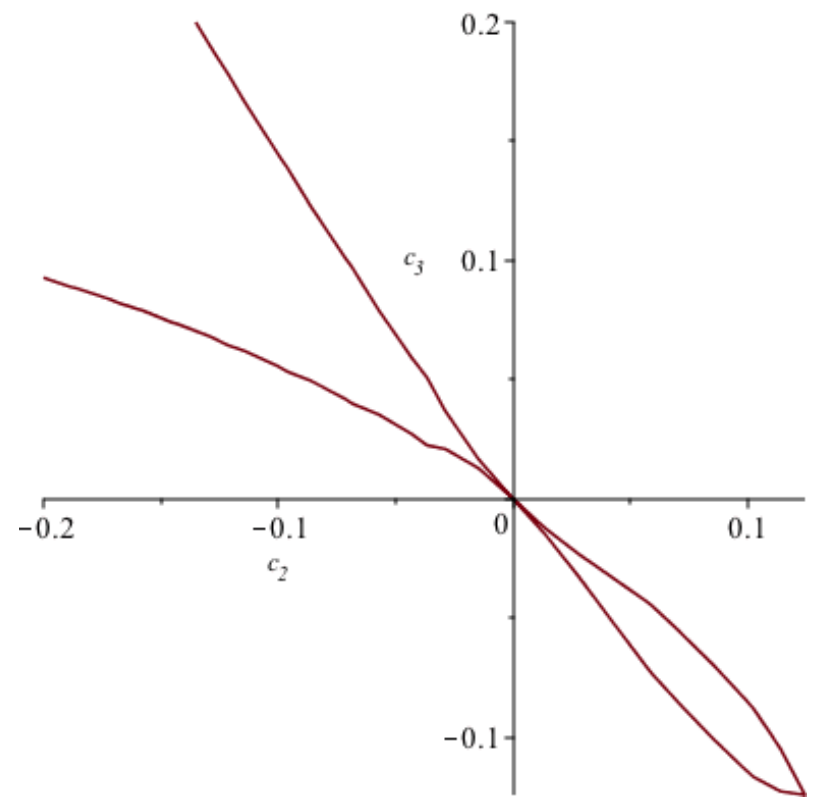

Fig. 1. The integrability condition of $\Psi \mathcal{D O}\left(S^{1}\right)$.

\subsection{The integrability condition}

The main result of this section is the following theorem.

Theorem 5.3. The infinitesimal deformation (5.1) corresponds to a polynomial deformation, if and only if the following (quartic) relation is satisfied:

$$
8 c_{2}^{3}+9 c_{3}^{2}+8 c_{2}^{2}+18 c_{2} c_{3}=0
$$

The integrability condition is represented in Fig. 1.

Let us first prove that the condition (2.2) is necessary for integrability of infinitesimal deformations.

Remark 5.4. In a cohomological interpretation, the obstructions to integrability of an infinitesimal deformation (5.1) which does not satisfy the condition (5.2), corresponds to a nontrivial class of $H^{2}\left(\operatorname{Vect}\left(S^{1}\right), \mathfrak{a f f}(1), \mathcal{F}_{5}\right)$.

Proof. The infinitesimal deformation (5.1) is clearly of the form:

$$
\tilde{\pi}(f \partial)=f \xi+c_{2} f^{\prime \prime} \xi^{-1}+c_{3} f^{\prime \prime \prime} \xi^{-2}+\cdots,
$$

where ... means the terms in $\xi^{-3}, \xi^{-4}, \ldots$ To compute the obstructions for integration of the infinitesimal deformation (5.1), one has to add the first nontrivial 
terms and impose the homomorphism condition. So, put

$$
\bar{\pi}(f \partial)=f \xi+c_{2} f^{\prime \prime} \xi^{-1}+c_{2} f^{\prime \prime \prime} \xi^{-2}+\Pi_{4}(c) f^{(i v)} \xi^{-3}+\Pi_{5}(c) f^{(v)} \xi^{-4},
$$

where $\Pi_{4}(c)$ and $\Pi_{5}(c)$ are some polynomials in $c=\left(c_{2}, c_{3}\right)$ and compute the difference. Collecting the terms in and yields:

$$
\begin{gathered}
2 \Pi_{4}(c)=-c_{2}^{2}-3 c_{3}-c_{2}, \\
5 \Pi_{5}(c)=-c_{2} c_{3}+c_{2}^{2}-6 \Pi_{4}(c)+c_{2} .
\end{gathered}
$$

Let us go one step further, expand our deformation up to $\xi^{-6}$, that is, put

$$
\overline{\bar{\pi}}(f \partial)=\bar{\pi}(f \partial)+\Pi_{6}(c) f^{(v i)} \xi^{-5}
$$

the homomorphism condition leads to a nontrivial relation for the parameters:

$$
10\left(c_{2} c_{3}-c_{2}^{2}-c_{2}-10 \Pi_{5}-c_{2}-c_{2} \Pi_{4}\right)=18\left(3 c_{2} \Pi_{4}+3 c_{2} c_{3}-2 c_{3}^{2}+10 \Pi_{4}+5 c_{3}\right) .
$$

Substituting the expressions (5.5) and (5.6) for $\Pi_{4}$ and $\Pi_{5}$, one gets the formula (5.2), we have thus shown that this condition is necessary for integrability of infinitesimal deformations.

This completes the proof of Theorem 5.3.

\subsection{Introducing the parameter $h$}

\subsubsection{Contraction of the Lie algebra $\Psi \mathcal{D O}\left(S^{1}\right)$}

The contraction is invented by E. Wigner and I. Inonu in the fifties, then developed by some physicists.

If $\mathfrak{g}$ is a Lie algebra, consider a one parameter group $\Phi_{t}: \mathfrak{g} \rightarrow \mathfrak{g}$ of linear endomorphisms, such that $\Phi_{t}$ be an isomorphism for $t \neq 0, \Phi_{1}=i d$ and $\Phi_{0}$ be singular. One defines the bracket $[,]_{t}$ on $\mathfrak{g}$ by the formula

$$
[X, Y]_{t}=\Phi_{t}^{-1}\left[\Phi_{t}(X), \Phi_{t}(Y)\right]
$$

For $t=1$, it is the bracket of $\mathfrak{g}$, and it is isomorphic to $[,]_{t}$ for $t \neq 0$. Suppose that for all $X$ and $Y$, the limit: $[X, Y]_{0}=\lim _{t \rightarrow 0}[X, Y]_{t}$ exist, then $[,]_{0}$ is a Lie bracket on $\mathfrak{g}$ not isomorphic to the previous one because $\Phi_{0}$ is singular. The algebra $\mathfrak{g}$ provided with the bracket [, ] called the contracted Lie algebra of $\mathfrak{g}$ by $\Phi_{t}$.

Now, let us consider the map $\Phi_{h}: \Psi \mathcal{D O}\left(S^{1}\right) \rightarrow \Psi \mathcal{D O}\left(S^{1}\right)$ defined by $\Phi_{h}\left(a \partial^{p}\right)=$ $a h^{p} \partial^{p}$, a straightforward computation show that

$$
\Phi_{h}^{-1}\left(\left[\Phi_{h}(A), \Phi_{h}(B)\right]\right)=h\left(\frac{\partial A}{\partial \partial} \frac{\partial B}{\partial x}-\frac{\partial B}{\partial \partial} \frac{\partial A}{\partial x}\right)+h O(h) .
$$

The limit exists and we have $\lim \frac{1}{h}[A, B]_{h}=\{A, B\}$ for $h \rightarrow 0$. Here, $\{A, B\}$ means the Poisson bracket on the symbols $(x, \partial)$ views this time as a function of two variables. So, we used the contraction of the Lie algebra $\Psi \mathcal{D O}\left(S^{1}\right)$ on the Poisson algebra $\left.P=C^{\infty}\left(S^{1}\right)\left[\partial, \partial^{-1}\right]\right]$ equipped with the canonical bracket. 


\subsubsection{Contraction of $\mathfrak{h}$-trivial deformations}

Given a $\mathfrak{h}$-trivial deformation of one of the types, (3.5) or (3.2), one can modify it so that it becomes a deformation with values in the deformed algebra $\operatorname{\Psi DO}_{h}\left(S^{1}\right)$. Namely, if one sets $\pi_{t}^{h}=\Phi_{h}^{-1} \circ \tilde{\pi}_{t}$, then one has

$$
\pi_{t}^{h}([F, G])=\Phi_{h}^{-1}\left(\left[\tilde{\pi}_{t}(F), \tilde{\pi}_{t}(G)\right]\right)=\left[\pi_{t}(F), \pi_{t}(G)\right]_{h}
$$

and so $\pi_{t}^{h}: \operatorname{Vect}\left(S^{1}\right) \rightarrow \Psi \mathcal{D O} \mathcal{O}_{h}\left(S^{1}\right)$ is a $\mathfrak{h}$-trivial (formal) deformation of the standard embedding.

\subsubsection{Parametrization: The integrability condition}

One can now modify the relation in order to get a deformation in $\Psi \mathcal{D O} \mathcal{O}_{h}\left(S^{1}\right)$, the scalar $h$ then appears with different powers according to the weight of the successive terms in the formula (5.2). One gets finally:

\section{Proposition 5.5. This relation}

$$
8 c_{2}^{3}+9 c_{3}^{2}+h\left(18 c_{2} c_{3}\right)+h^{2} 8 c_{2}^{2}=0
$$

is a necessary integrability condition for infinitesimal deformations (5.1) in $\Psi \mathcal{D O}_{h}\left(S^{1}\right)$. The integrability condition is represented on Fig. 2.

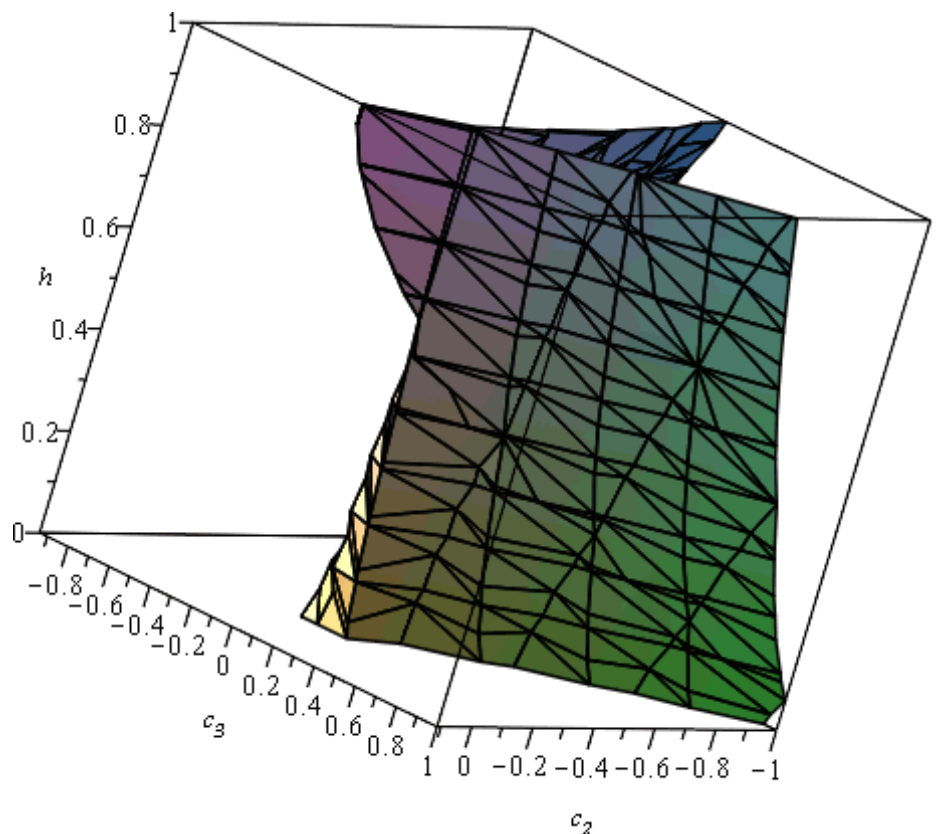

Fig. 2. The integrability condition of $\Psi \mathcal{D O} \mathcal{O}_{h}\left(S^{1}\right)$. 
Now, let us change the parameters $c_{2}$ and $c_{3}$ and rewrite the expression (5.7) in a canonical form. One checks by an elementary straightforward calculation the

Proposition 5.6. For all $\sigma \in \mathbb{C}$, the constants

$$
\begin{aligned}
& c_{2}=-\frac{\sigma(\sigma+h)}{2}, \\
& c_{3}=\frac{\sigma^{2}(h-\sigma)}{2}+\frac{\sigma(h-\sigma)(2 h-\sigma)}{6}
\end{aligned}
$$

satisfy the relation (5.7).

Any couple $c_{2}, c_{3}$ satisfying the relation (5.7) is of the form (5.8) and (5.9), for some $\sigma \in \mathbb{C}$.

Proof. By direct computation.

Proposition 5.7. Every infinitesimal afff(1)-trivial deformation of the standard embedding of $\operatorname{Vect}\left(S^{1}\right)$ into $\mathcal{P}$ is equivalent to:

$$
f \partial \mapsto f \xi+c_{1} f^{\prime \prime} \xi^{-1}+c_{2} f^{\prime \prime \prime} \xi^{-2}
$$

where $c_{1}, c_{2} \in \mathbb{R}($ or $\mathbb{C})$ are parameters.

Theorem 5.8. An infinitesimal afff(1)-trivial deformation (5.10) corresponds to a polynomial deformation of the standard embedding $\operatorname{Vect}\left(S^{1}\right) \rightarrow \mathcal{P}$, if and only if it satisfies the following condition:

$$
8 c_{1}^{3}+9 c_{2}^{2}=0
$$

Proof. For, $h=0$ in (5.7), one gets the polynomial we have obtained in the semiclassical (Poisson) case.

\section{6. $\mathfrak{s l}(2)$-Trivial Deformation of the $\operatorname{Vect}\left(S^{\mathbf{1}}\right)$}

In this section, we are interested to the case of the $\mathfrak{s l}(2)$-trivial deformation. Let $\rho_{0}: \operatorname{Vect}\left(S^{1}\right) \rightarrow \Psi \mathcal{D O}$ be an embedding of Lie algebras. A formal deformation of $\rho_{0}$ is a formal series

$$
\rho(t)=\rho_{0}+\sum_{i=1}^{m} t_{i} \Theta_{i}+\sum_{i, j=1}^{m} t_{i} t_{j} \rho_{i j}^{(2)}+\cdots,
$$

where the $\Theta_{i}, \rho_{i j}^{(2)}, \ldots$ are linear $\mathfrak{s l}(2)$-relative maps from $\operatorname{Vect}\left(S^{1}\right)$ to $\Psi \mathcal{D O}$ with the map

$$
\rho(t): \operatorname{Vect}\left(S^{1}\right) \rightarrow \mathbb{C}\left[\left[t_{1}, \ldots, t_{m}\right]\right] \otimes \Psi \mathcal{D O}
$$

which satisfies the homomorphism condition. 


\section{Corollary 6.1.}

$$
\mathrm{H}^{1}\left(\operatorname{Vect}\left(S^{1}\right), \mathfrak{s l}(2), \Psi \mathcal{D} \mathcal{O}_{h}\right)=\mathbb{R} .
$$

The nontrivial cocycles generating the relative cohomology groups are as follows:

$$
\Theta_{3}(f \partial)=\sum_{n=2}^{\infty}(-1)^{n} \frac{3(n-1)}{n+1} f^{(n+1)} \xi^{-n} .
$$

It is clear that the same result holds for $\mathcal{P}$ :

$$
\mathrm{H}^{1}\left(\operatorname{Vect}\left(S^{1}\right), \mathfrak{s l}(2), \mathcal{P}\right)=\mathbb{R} .
$$

The nontrivial cocycles generating the relative cohomology groups are as follows:

$$
C_{2}(f \partial)=f^{\prime \prime \prime} \xi^{-2}
$$

The main result of this subsection is the following.

\section{Theorem 6.2.}

(1) Any formal $\mathfrak{s l}(2)$-trivial deformation of the standard embedding of $\operatorname{Vect}\left(S^{1}\right)$ into $\Psi \mathcal{D O}$ is equivalent to its infinitesimal part:

$$
f \partial \mapsto f \xi+c_{3} \Theta_{3}(f \partial),
$$

where $c_{3} \in \mathbb{R}($ or $\mathbb{C})$ is parameter.

(2) Any formal $\mathfrak{s l}(2)$-trivial deformation of the standard embedding of $\operatorname{Vect}\left(S^{1}\right)$ into $\mathcal{P}$ is equivalent to its infinitesimal part:

$$
f \partial \mapsto f \xi+c_{3} C_{3}(f \partial),
$$

where $c_{3} \in \mathbb{R}($ or $\mathbb{C})$ is parameter.

Proof. Consider a formal deformation of the embedding (3.2):

$$
\widetilde{\rho}=\rho_{0}+c_{3} \Theta+\sum_{m \geq 2} c^{m} \rho^{(m)},
$$

where the highest-order terms $\rho^{(m)}$ are linear $\mathfrak{s l}(2)$-relative maps from $\operatorname{Vect}\left(S^{1}\right)$ to $\Psi \mathcal{D O}$.

By a direct computation, we check that $\llbracket \Theta_{3}, \Theta_{3} \rrbracket=0$. The solution $\rho_{i, j}^{(2)}$ of $(3.9)$ is defined up to a 1-cocycle and it has been shown in $[1,2,7-10,14-16]$ that different choice of solutions of (3.9) corresponds to equivalent deformations. Thus, one can always kill $\rho_{i, j}^{(2)}$. Then, by recurrence, the highest-order terms satisfy the equation $\delta \rho_{i, j}^{(m)}=0$ and can also be killed.

As before, we prove the result concerning the deformation of the standard embedding of $\operatorname{Vect}\left(S^{1}\right)$ into $\mathcal{P}$ from the fact that we have by a direct computation $\llbracket C_{3}, C_{3} \rrbracket=0$. 


\section{Conclusion and Further Remarks}

\subsection{Conclusion}

In this paper, we study the $\mathfrak{h}$-Trivial deformations of the standard embedding of the Lie algebra $\operatorname{Vect}\left(S^{1}\right)$ of smooth vector fields on the circle, into the Lie algebra of functions on the cotangent bundle $T^{*} S^{1}$. We classify the deformations of this action that become trivial once restricted to $\mathfrak{h}$, where $\mathfrak{h}=\mathfrak{a f f}(1)$ or $\mathfrak{s l}(2)$. We prove that these conditions are also sufficient.

\subsection{Further remarks}

It would be interesting in the analog super structures: it would be interesting to study the $\mathfrak{k}$-trivial deformation of the standard embedding of the Lie superalgebra $\mathcal{K}(1)$ of smooth vector fields on the supercircle $S^{1 \mid 1}$.

Note that a Lie superalgebra is a generalization of a Lie algebra to include a $\mathbb{Z}_{2}$-grading. Lie superalgebras are important in theoretical physics where they are used to describe the mathematics of super symmetry. Namely, we consider the superspace $S^{1 \mid 1}$ equipped with the contact structure determined by a 1-form $\alpha$, and the Lie superalgebra $\mathcal{K}(1)$ of contact vector fields on $\mathbb{R}^{1 \mid 1}$.

\section{References}

[1] B. Agrebaoui, F. Ammar, P. Lecomte and V. Ovsienko, Multiparameter deformations of the module of symbols of differential operators, Int. Math. Res. Not. 16 (2002) 847-869.

[2] B. Agrebaoui, N. Ben Fraj, M. Ben Ammar and V. Ovsienko, Deformation of modules of differential forms, Nonlinear Math. Phys. 10(2) (2003) 148-156.

[3] I. Basdouri and M. Ben Ammar, Cohomology of osp (1|2) Acting on linear differential operators on the supercircle $S^{1 \mid 1}$, Lett. Math. Phys. 81 (2007) 239-251.

[4] I. Basdouri, M. Ben Ammar, N. Ben Fraj, M. Boujelbene and K. Kammoun, Cohomology of the Lie superalgebra of contact vector fields on $\mathbb{R}^{1 \mid 1}$ and deformations of the superspace of symbols, J. Nonlinear Math. Phys. 16(4) (2009) 1-37.

[5] I. Basdouri, M. Ben Ammar, B. Dali and S. Omri, Deformation of $\mathfrak{v e c t}(1)$-modules of symbols, doi: 10.1016/j.geomphys.2009.12.002.

[6] C. Conley, Conformal symbols and the action of contact vector fields over the superline, J. Reine Angew. Math. 633 (2009) 115-163.

[7] B. L. Feigin and D. B. Fuks, Homology of the Lie algebras of vector fields on the line, Funct. Anal. Appl. 14 (1980) 201-212.

[8] A. Fialowski, Deformations of Lie algebras, Math. USSR-Sb. 55 (1986) 467-473.

[9] A. Fialowski, An example of formal deformations of Lie algebras, in Deformation Theory of Algebras and Structures and Applications (Kluwer, 1988), pp. 375-401.

[10] A. Fialowski and D. B. Fuchs, Construction of miniversal deformations of Lie algebras, J. Funct. Anal. 161(1) (1999) 76-110.

[11] H. Gargoubi, N. Mellouli and V. Ovsienko, Differential operators on supercircle: Conformally equivariant quantization and symbol calculus, Lett. Math. Phys. 79 (2007) $51-65$.

[12] M. Gerstenhaber, On the deformation of rings and algebras I, II, III, Ann. Math. 79 (1964) 59-10; 84 (1966) 1-19; 88 (1968) 1-34. 
[13] A. Nijenhuis and R Richardson, Cohomology and deformations of algebraic structures, Bull. Amer. Math. Soc. 70 (1964) 406-411.

[14] A. Nijenhuis and P. W. Richardson Jr, Deformations of homomorphisms of Lie groups and Lie algebras, Bull. Amer. Math. Soc. 73 (1967) 175-179.

[15] V. Ovsienko and C. Roger, Deforming the Lie algebra of vector fields on $S^{1}$ inside the Lie algebra of pseudodifferential operators on $S^{1}$, AMS Transl. Ser. 2 (Adv. Math. Sci.) 194 (1999) 211-227.

[16] V. Ovsienko and C. Roger, Deforming the Lie algebra of vector fields on $S^{1}$ inside the Poisson algebra on $\dot{T}^{*} S^{1}$, Comm. Math. Phys. 198 (1998) 97-110. 